\title{
Etude De Quelques Proprietes Biologiques De Ocimum Gratissimum L., Une Lamiaceae Recoltee A Daloa (Côte d'Ivoire)
}

\section{Elisée Kporou Kouassi}

Laboratoire de Biochimie -Microbiologie

Université Jean Lorougnon Guédé Daloa, Côte d'Ivoire

\section{Sitapha Ouattara}

Laboratoire de Pharmacodynamie Biochimique, Université Félix Houphouët Boigny, Abidjan, Côte d'Ivoire

\section{Cendrine Seguin Sylvie Fournel Benoit Frisch}

Laboratoire de Conception et Application de Molécules Bioactives, Equipe Biovectorologie, UMR 7199 CNRS-Université de Strasbourg, Faculté de Pharmacie, Illkirch Cédex- France

Doi: 10.19044/esj.2018.v14n3p477 URL:http://dx.doi.org/10.19044/esj.2018.v14n3p477

\begin{abstract}
Introduction: Ocimum gratissimum is used in traditional Ivorian medicine for its multiple therapeutic virtues. Scope: Evaluate the biological properties of the essential oil of this plant. Methods: Antitumor activity was assessed using the MTS colorimetric assay on six (06) cell lines, the antifungal activity was evaluated by double dilution slant method on four (04) strains of clinical fungi, the potential antioxidant was determined by the DPPH test. Results: The strongest cytotoxic activity was obtained on Jurkat ( $T$ lymphoma) with an IC50 $=80 \mu \mathrm{g} / \mathrm{ml}$. The best antifungal activity was observed on C. albicans with MIC and FMC values obtained at $12.5 \mu \mathrm{g} / \mathrm{ml}$. Antioxidant activity was evaluated at $\mathrm{F}=187 \pm 1.57 \mathrm{mM}$ Trolox $/ \mathrm{ml}$ or $(\mathrm{I}=$ $38 \pm 0.74 \%$ ). Conclusion: The essential oil has interesting biological properties like as cytotoxic, antifungal and antioxidant.
\end{abstract}

Keywords: Ocimum gratissimum, cytotoxic, antifungal, antioxidant 


\section{Resume}

Introduction: Ocimum gratissimum est utilisée en médecine traditionnelle ivoirienne pour ses multiples vertus thérapeutiques. Objectif: Evaluer les propriétés biologiques de l'huile essentielle de cette plante. Méthodologie: L'activité antitumorale a été évaluée au moyen du test colorimétrique MTS sur six (06) lignées cellulaires, l'activité antifongique a été évaluée par la méthode de la double dilution en tubes inclinés sur quatre (04) souches de champignons cliniques, le potentiel antioxydant a été déterminée par le test au DPPH. Résultats: L'activité cytotoxique la plus forte a été obtenue sur Jurkat (lymphome T) avec une $\mathrm{CI}_{50}=80 \mu \mathrm{g} / \mathrm{ml}$. La meilleure activité antifongique a été observée sur $C$. albicans avec des valeurs de CMI et $\mathrm{CMF}$ obtenues à $12,5 \mu \mathrm{g} / \mathrm{ml}$. L'activité antioxydante a été évaluée à $\mathrm{F}=187$ $\pm 1,57 \mathrm{mM}$ Trolox/ml ou ( $\mathrm{I}=38 \pm 0,74 \%)$. Conclusion: Cette huile essentielle possède d'intéressantes propriétés biologiques à savoir cytotoxique, antifongique et antioxydante.

Mots-Clefs: Ocimum gratissimum, cytotoxique, antifongique, antioxydante

\section{Introduction}

Depuis l'antiquité, les hommes utilisaient les huiles essentielles autant pour leurs besoins cosmétiques, alimentaires que thérapeutiques (Heath, 1981; Robert, 2000). De nos jours, de nombreux composés volatiles sont aujourd'hui des ingrédients courants des préparations pharmaceutiques. Le thymol, par exemple, est employé en soins dentaires pour ses propriétés antiseptiques ou encore l'eugénol pour ses propriétés analgésiques (Pauli, 2001). Pour tenter de trouver de nouveaux remèdes aux fléaux actuels, la communauté scientifique s'est récemment tournée vers les constituants des huiles essentielles, car un nombre non négligeable de composés volatiles, tels que les sesquiterpènes, ont montré des activités pharmacologiques remarquables contre les maladies comme le cancer (Modzelewska et al., 2005). En outre, il a été démontré que la présence des radicaux libres dans l'organisme est un facteur de risque pour la progression de certaines maladies telles que le cancer, le diabète, l'alzheimer, l'artériosclérose ainsi que le vieillissement (Gardner, 1997; Butterfield, 2002, Kohen et Nyska, 2002). Ces radicaux libres conduisent à des mutations de gênes qui peuvent entrainer des perturbations de la transcription et la traduction du signal intracellulaire à l'origine de la cancérogenèse (Kouamé et al, 2009; Valko et al.,2006; Mashtaq et al, 2013). A ce sujet, Owen et al., (2000) ont également montré que les extraits végétaux riches en composés phénoliques et présentant un pouvoir antioxydant très marqué pourraient jouer un rôle intéressant dans la prévention du cancer car ce sont des stabilisateurs des radicaux libres. 
Chez les immunodéprimés, particulièrement chez les personnes vivant avec le VIH (PPV), les affections fongiques constituent l'une des principales causes de décès à travers le monde du fait du nombre limité de molécules antifongiques, de leur coût très souvent élevé et surtout de l'émergence de souches multirésistantes aux antifongiques usuels (Rex et al., 1995; Sheelan et al., 1999; Young et al., 1999). La découverte de nouvelles molécules permettrait; à défaut d'éliminer les facteurs pathogéniques, d'améliorer l'état de santé des malades. Pour ce faire, le règne végétal base de notre médecine traditionnelle est susceptible de fournir un grand nombre de molécules douées de propriétés biologiques intéressantes (Newman et al., 2003; Dzoyem et al., 2006). C'est dans cette optique que la présente étude a été menée en vue d'étudier in vitro le potentiel cytotoxique; antifongique et antioxydant de l'huile essentielle de Ocimum gratissimum L, une Lamiaceae récoltée dans la ville de Daloa (Côte d'Ivoire) et largement utilisée en médecine traditionnelle ivoirienne pour ses nombreuses vertus thérapeutiques.

\section{Matériel et Méthodes}

\section{Collection et identification de la plante}

Ocimum gratissimum L., est une plante de la famille des lamiaceae. Elle a été récoltée à Daloa dans la région du haut Sassandra en Côte d'Ivoire en Février 2016. Des échantillons de cette plante ont été acheminés au Centre National de Floristique (CNF) de l'université Félix Houphouët Boigny pour être identifiés au spécimen CNF 304.

\section{Lignées cellulaires et milieux de culture}

Toutes les lignées cellulaires de cancers humains utilisées dans cette étude proviennent de American Type Culture Collection (ATCC) et ont été mises à notre disposition par l'équipe Biovectorologie de l'UMR 7199 Université de Strasbourg-CNRS. Il s'agit des lignées cellulaires en suspension (Ramos CRL 1596 (lymphome B), Jurkat (Lymphome T)) et adhérentes (U87 HTB-14 (glioblastome), HCT 116 CCL 47 (colocarcicome du colon), MDAMB-231 (cancer du sein), HeLa (cancer du col de l'utérus)). Les milieux de culture étaient composés de RPMI 1640 (Roswell Park Memorial Institute) AQ media et une solution 10\% SVF (Sérum de Veau Fœtal) decomplémenté. Les cellules sont soit adhérentes, c'est à dire qu'elles se fixent au fond de la flasque et se multiplient donc sur une surface, soit en suspension dans leur milieu de culture.

\section{Les champignons et milieux de culture}

Le support microbien est composé de champignons de souche clinique dont deux levures Candida albicans My 03812 417/P et Candida glabrata My 0284 20/D et deux moisissures Aspergillus flavus 1006/AB et Aspergillus 
fumigatus 896/AB. Les Levures ont ont été fournies par le Laboratoire de Mycologie de l'Institut pasteur Abidjan et les moisissures provenaient du département de Mycologie de l'UFR des Sciences Médicales de l'Université Félix houphouët Boigny. Les cultures des champignons ont été réalisées sur milieu de culture SABOURAUD AGAR (BIO RAD 64494, lot 6J2218).

\section{Extraction de l'huile essentielle}

La technique d'extraction des huiles essentielles (HE) utilisée dans cette étude est l'hydrodistillation. Celle-ci a été réalisée à partir de $500 \mathrm{~g}$ de feuilles fraîches de Ocimum gratissimum (Lamiaceae) à l'aide d'un extracteur de type Clevenger pendant 3 heures au Laboratoire de Chimie de l'Université Jean Lorougnon Guédé Daloa en Côte d'Ivoire. Les essences sont recueillies dans des flacons qui sont recouverts de papier aluminium afin de les préserver de tout effet négatif de la lumière. Les flacons ont été par la suite conservés au réfrigérateur à la température de $12^{\circ} \mathrm{C}($ Celso et $a l ., 1999)$. L' huile essentielle de $O$. gratissimum a été testée pour ses activités antitumorale, antioxydante et antifongique.

\section{Evaluation de l'activité cytotoxique}

L'activité cytotoxique ou anticancéreuse a été faite par le moyen du test colorimétrique MTS [3-(4,5-diméthylthiazol-2-yl)-(3carboxyméthoxyphényl)-2-(4-sulfophényl)-2H-tétrazolium] développée par Promega, le CellTiter96 AQueous One Solution Cell Proliferation Assay. Il s'agit d'un test in vitro mesurant la prolifération des cellules. Cette méthode est basée sur la réduction d'un réactif le MTS en un produit coloré, le formazan, par la NADPH ou NADH déshydrogénase des cellules métaboliquement actives (Figure 1).<smiles>Cc1nc(N2NC(c3cccc(OCC(=O)O)c3)=NN2c2ccc(S(=O)(=O)[O-])cc2)sc1C</smiles>

MTS<smiles>Cc1nc(N=NC(=NN(C)c2ccc([S-])cc2)c2cccc(OCC(=O)[O-])c2)sc1C</smiles>

Formazan

Figure 1 : La réduction du MTS en formazan.

La solution-mère de l'huile essentielle a été préparée dans le Diméthylsulfoxide 1\% (DMSO 1\%) puis les différentes concentrations à tester 
sont faites par une série de dilutions en cascades au 1/2 donnant une gamme de concentrations de $1 \mathrm{mg} / \mathrm{ml}$ à $0,007 \mathrm{mg} / \mathrm{ml}$. Les cultures cellulaires ont été faites sous un Poste de Sécurité Microbiologique (PSM) afin d'éviter les contaminations bactériennes ou fongiques. Les cellules sont ensemencées dans des plaques 96 puits de 15000 à 30000 cellules/puits (Tableau I). Elles sont cultivées pendant 4 heures dans leur milieu de culture puis traitées par les différentes concentrations extemporanément préparées de l'huile essentielle de Ocimum gratissimum

Après 24 heures d'incubation à $37^{\circ} \mathrm{C}, 5 \% \mathrm{CO} 2$ et $95 \%$ humidité, les cellules sont observées au microscope inversé puis mises en contact avec 20 $\mu \mathrm{L}$ d'une solution de MTS. Après 2 heures d'incubation, l'absorbance est mesurée grâce à un lecteur de microplaques ELx808 de marque Biotek à une longueur d'onde de $490 \mathrm{~nm}$ (avec une référence de $700 \mathrm{~nm}$ ). La quantité de formazan produite est directement proportionnelle au nombre de cellules vivantes (Owen et al., 2000). Chaque expérience est réalisée en triplicate pour chaque concentration de l'huile testée. Les tests ont été effectués avec l'huile essentielle dissoute dans le DMSO 1/\%. Pour chaque test, un contrôle au DMSO est réalisé pour vérifier qu'à lui seul il ne tue pas les cellules.

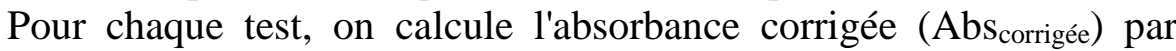
soustraction de la valeur de l'absorbance à $700 \mathrm{~nm}$ à celle de $490 \mathrm{~nm}$ car elle correspond aux cellules ou aux débris de cellules. Puis on calcule le ratio : en divisant notre valeur Abs corrigée par la moyenne des puits contrôles, c'est-àdire des puits qui ne contiennent que des cellules et n'ont pas été stimulées avec l'huile (Figure 2). A partir des ratios, des graphiques sont construits et la concentration inhibitrice $50 \%\left(\mathrm{CI}_{50}\right)$ de l'huile pour chaque lignée cellulaire est déterminée. Cette mesure quantitative indique la concentration de l'huile de $O$. gratissimum nécessaire pour inhiber la moitié de la prolifération cellulaire globale par rapport à un contrôle n'ayant subi aucun traitement. Les résultats sont présentés sous la forme : moyenne \pm ESM (Ecart Type à la Moyenne).

Tableau I: Concentrations des cellules à ensemencer en fonction de la lignée cellulaire

\begin{tabular}{|c|c|c|c|c|c|c|}
\hline Lignées & HCT116 & Hela & Jurkat & $\begin{array}{c}\text { MDA- } \\
\text { MDB- } \\
231\end{array}$ & $\begin{array}{c}\text { Ramos } \\
\text { CRL } \\
1596\end{array}$ & $\begin{array}{c}\text { U87 } \\
\text { HTB- } \\
14\end{array}$ \\
\hline $\begin{array}{c}\text { Ensemencement } \\
\text { (Cellules/puits) }\end{array}$ & 30000 & 25000 & 30000 & 40000 & 30000 & 30000 \\
\hline
\end{tabular}




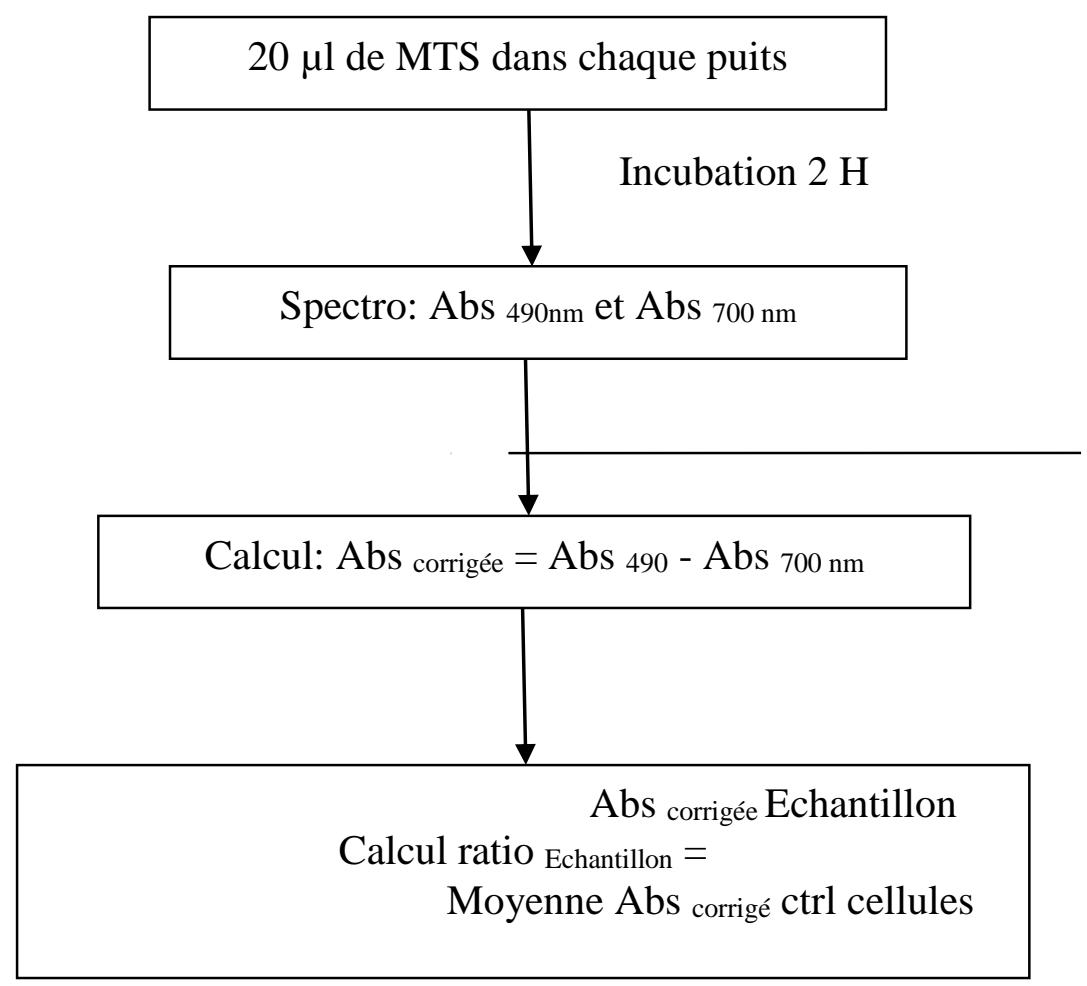

Figure 2 : Schéma du principe de révélation au MTS

\section{Evaluation de l'activité antioxydante}

L'activité antioxydante a été évaluée par la mesure de la possibilité de reduction des antioxdants en présence du DPPH (2, 2' diphenyl-1picrylhydrazyl) [18]. Dans ce test, les antioxydants réduisent le DPPH ayant une couleur violette en un composé jaune, dont l'intensité de la couleur mesurée à $515 \mathrm{~nm}$ à l'aide d'un spectrophotomètre est inversement proportionnelle à la capacité des antioxydants présents dans le milieu à donner des protons (Bouchet et al., 1998; Sanchez-Moreno, 2002). La mesure de l'activité antioxydante pourrait nous aider à comprendre le potentiel cytotoxique de l'huile essentielle de cette plante. L'expression de l'activité antioxydante de l'huile essentielle de Ocimum gratissimum a été faite en prenant comme solution de reférence standard le (S)-(-)-6-hydroxy-2,5,7,8tetramethylchroman-2- acide carboxylique, appelé Trolox (T). le pourcentage d'inhibition (I\%) a été calculée en utilisant la formule suivante:

$$
\mathrm{I} \%=\left[\left(\mathrm{A}_{\mathrm{B}}-\mathrm{A}_{\mathrm{A}}\right) / \mathrm{A}_{\mathrm{B}}\right] \mathrm{X} 100
$$

dans laquelle: $A_{B}=$ absorbance de la solution standard; $A_{A}=$ absorbance de l'échantillon $(\mathrm{t}=30 \mathrm{~min})$.

Brièvement, $80 \mu \mathrm{M}$ de DPPH a été dissout dans de l'éthanol pur à $98 \%$. Le mélange a été vigoureusement agité et laissé à l'obscurité à la température 
de la salle à $25^{\circ} \mathrm{C}$ pendant $10 \mathrm{mn}$ et la décoloration par rapport au contrôle négatif contenant uniquement la solution de DPPH est mesurée à $515 \mathrm{~nm}$. Un volume de $250 \mu \mathrm{l}$ de l'échantillon avec $1,45 \mathrm{ml}$ de solution standard a été ajouté dans chaque puits de la plaque multipuits de 96 puits. Les résultats sont exprimés en mM Trolox par gramme. Les mesures ont été faites 3 fois et l'huile essentielle a été utilisée à une concentration $0,1 \mathrm{~g}$ sample/1 $\mathrm{ml}$ de solvant (Sanchez-Moreno, 2002; Kim et al., 2003).

\section{Etude de l'activité antifongique de l'huile essentielle de $O$. gratissimum}

L'huile essentielle de $O$. gratissimum a été diluée dans une quantité minimale de DMSO 1\% afin d'obtenir une émulsion homogène avec le milieu de culture Sabouraud. Tous les tests antifongiques ont été réalisés sur milieu solide par la méthode de la double dilution en tubes inclinés. Les concentrations de l'huile essentielle variaient de $100 \mu \mathrm{g} / \mathrm{ml}$ à $1,562 \mu \mathrm{g} / \mathrm{ml}$. Les essais antimicrobiens ont été réalisés par l' ensemencement en stries transversales (jusqu'à épuisement) de $10 \mu \mathrm{l}$ de la suspension $10^{-1}$ de l'inoculum préalablement préparé. Cela correspond à 1000 cellules ensemencées. Les cultures ainsi réalisées ont été incubées à $30^{\circ} \mathrm{C}$. Après 48 heures d'incubation, les colonies de chaque germe cible ont été dénombrées. La croissance dans les tubes expérimentaux de chaque série a été évaluée en pourcentage de survivance, par rapport à $100 \%$ de survivance dans le tube témoin de contrôle de croissance (Ackah, 2004; Kporou et al., 2010). Pour chaque test, un contrôle au DMSO 1\% est réalisé pour vérifier qu'à lui seul il ne tue pas les cellules.

\section{Détermination des paramètres antifongiques (CMI, CMF)}

La CMI (Concentration Minimale Inhibitrice) est la plus petite concentration d'extrait à partir de laquelle on n'observe aucune croissance visible à l'œil nu dans les tubes tests. La CMF a été déterminée successivement à la CMI. Brièvement, le contenu de chaque tube, allant de la valeur de la CMI aux concentrations les plus élevées a été ensemencée par strie à la surface de la gélose Sabouraud neuve coulée dans des tubes et incubés à $30^{\circ} \mathrm{C}$ pendant 48h. La croissance microbienne dans ces différents tubes ensemencés a été comparée à celle de la dilution $10^{0}$ à $10^{-4}$ de la suspension mère, à la recherche de la croissance laissant $0,01 \%$ de survivants (dilution $10^{-4}$ ). La concentration du tube dans lequel le nombre de colonies est identique à celui de la dilution $10^{-4}$ correspond à la Concentration Minimale Fongicide (CMF). Les valeurs de CMI et CMF permettront de préciser si notre huile essentielle est fongistatique ou fongicide (Kporou et al., 2010; Djeneb et al., 2016). 


\section{Résultats}

La visualisation de l'activité cytotoxique sur des cellules HCT 116 a été illustrée par la figure 3. Les valeurs des $\mathrm{CI}_{50}$ après l'évaluation de l'activité cytotoxique de l'huile essentielle de Ocimum gratissimum sur chaque lignée cellulaire ont été présentées dans le Tableau II.

Les résultats ont montré que l'huile essentielle de Ocimum gratissimum a une activité cytotoxique dose dépendante sur les lignées cellulaires HCT 116, Hela, Jurkat, MDA-MDB-231, Ramos CRL 1596 et U87 HTB-14. La plus petite valeur de $\mathrm{CI}_{50}$ a été obtenue à $80 \mu \mathrm{g} / \mathrm{ml}$ sur la lignée Jurkat alors que la plus grande valeur fut déterminée sur la lignée U87 HTB-14 à $200 \mu \mathrm{g} / \mathrm{ml}$. Les figures $4,5,6,7,8$ et 9 correspondent à l'effet cytotoxique de l'huile OG dissous dans du DMSO et le DMSO seul sur les lignées dans les puits. Les résultats sont une moyenne de 3 manipulations. Le graphique «DMSO » correspond à un contrôle, qui permet de vérifier que le DMSO seul ne tue pas la lignée cellulaire cible. En présence de DMSO seul, les ratios calculés sont presque constants autour de 1 , et même avec la plus grande concentration de DMSO. Cependant, les courbes de l'huile $\mathrm{OG}+\mathrm{DMSO}$ décroissent au fur et à mesure que les concentrations augmentaient dans les puits.

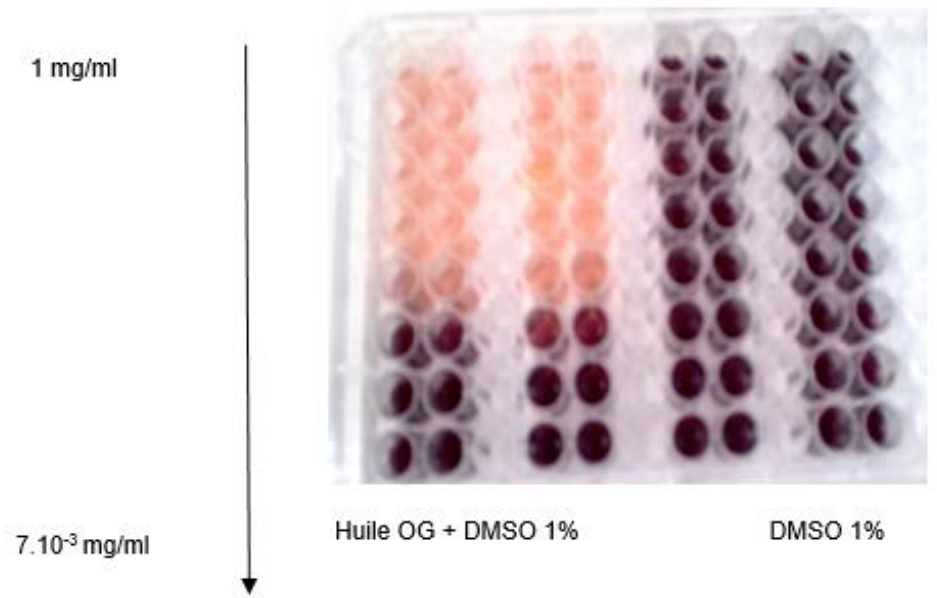

Figure 3: Illustration d'une plaque de 96 puits dans laquelle un test colorimétrique MTS a été réalisé en présence de l'huile essentielle OG+DMSO 1\% et DMSO 1\% sur les lignées cellulaires HCT 116 (Colocarcicome)

Tableau II: Valeurs des $\mathrm{CI}_{50}$ de l'Huile essentielle OG sur les différentes lignées

\begin{tabular}{|c|c|c|c|c|c|c|}
\hline $\begin{array}{c}\text { Lignées } \\
\text { Cellulaires }\end{array}$ & HCT116 & Hela & Jurkat & $\begin{array}{c}\text { MDA- } \\
\text { MDB-231 }\end{array}$ & $\begin{array}{c}\text { Ramos } \\
\text { CRL 1596 }\end{array}$ & $\begin{array}{c}\text { U87 } \\
\text { HTB-14 }\end{array}$ \\
\hline $\mathrm{CI}_{50}(\mu \mathrm{g} / \mathrm{ml})$ & $150 \pm 0,02$ & $\begin{array}{c}180 \pm \\
0,02\end{array}$ & $\begin{array}{c}80 \pm \\
0,02\end{array}$ & $150 \pm 0,02$ & $160 \pm 0,02$ & $200 \pm$ \\
& & & & 0,02 \\
\hline
\end{tabular}




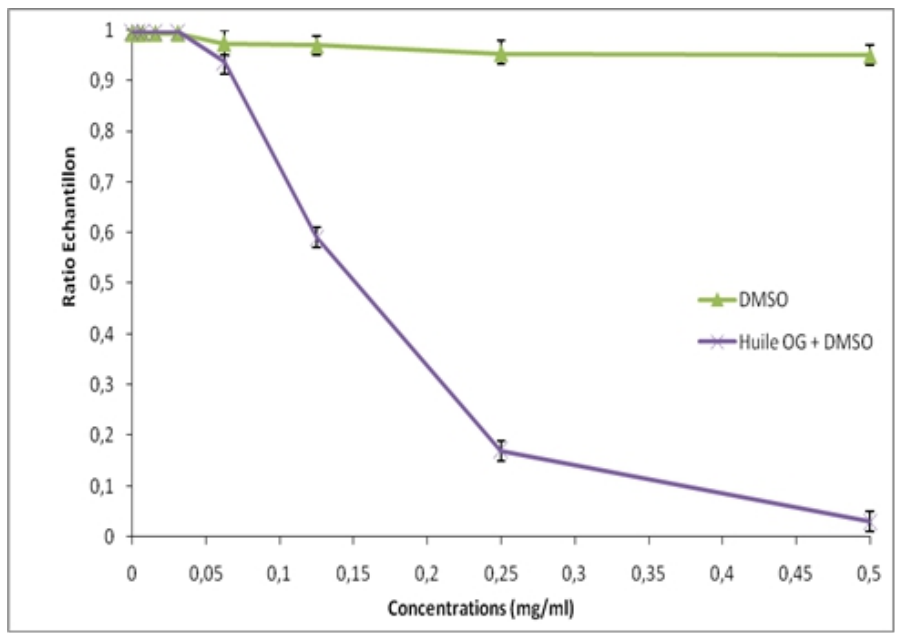

Figure 4: Effet cytotoxique dose-réponse de l'Huile essentielle OG solubilisé au DMSO et le contrôle DMSO sur la lignée HCT 116

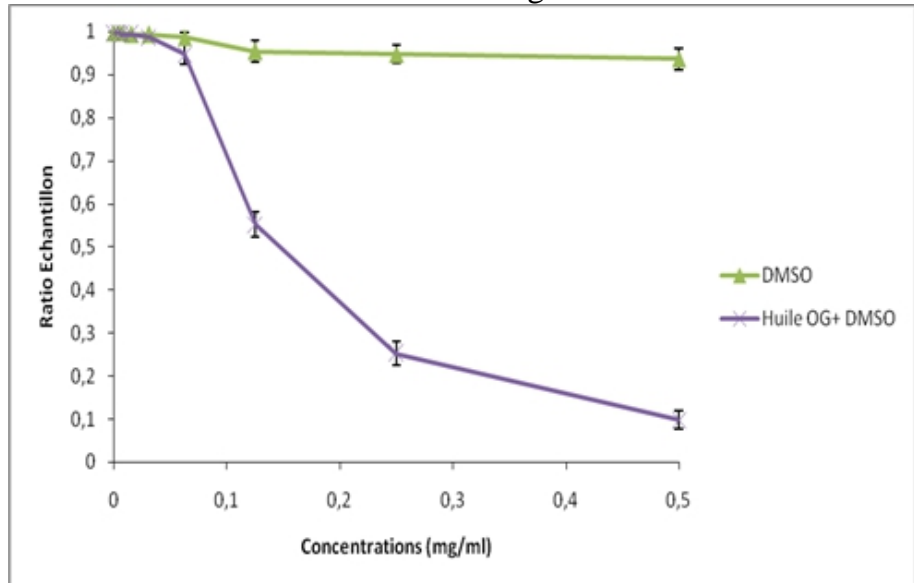

Figure 5: Effet cytotoxique dose-réponse de l'Huile essentielle OG solubilisé au DMSO et le contrôle DMSO sur la lignée Ramos

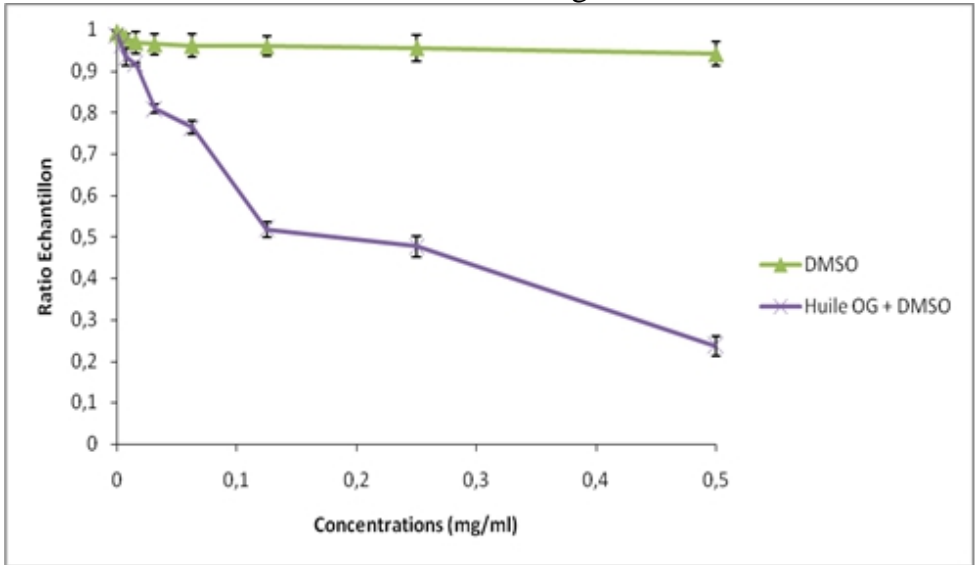

Figure 6: Effet cytotoxique dose-réponse de l'Huile essentielle OG solubilisé au DMSO et le contrôle DMSO sur la lignée Hela 


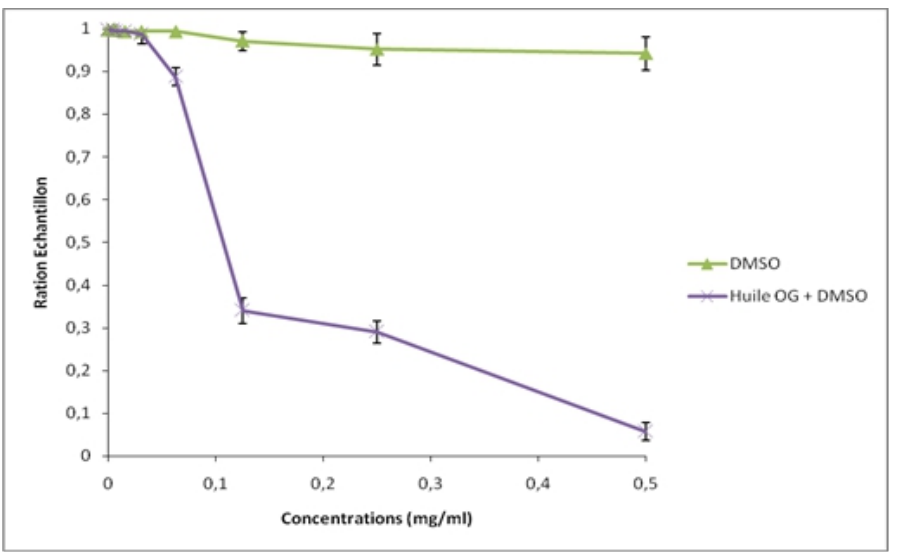

Figure 7: Effet cytotoxique dose-réponse de l'Huile essentielle OG solubilisé au DMSO et le contrôle DMSO sur la lignée Jurkat

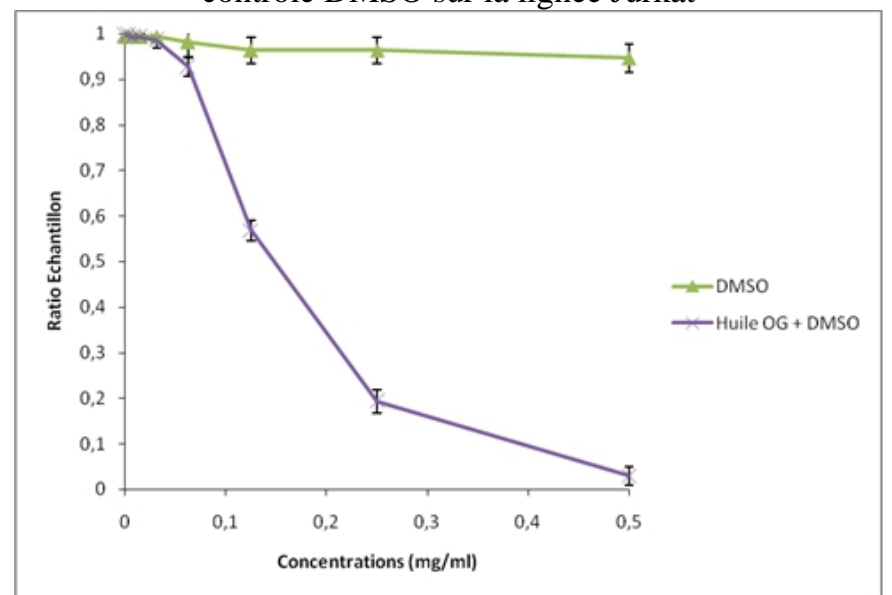

Figure 8: Effet cytotoxique dose-réponse de l'Huile essentielle OG solubilisé au DMSO et le contrôle DMSO sur la lignée MDA

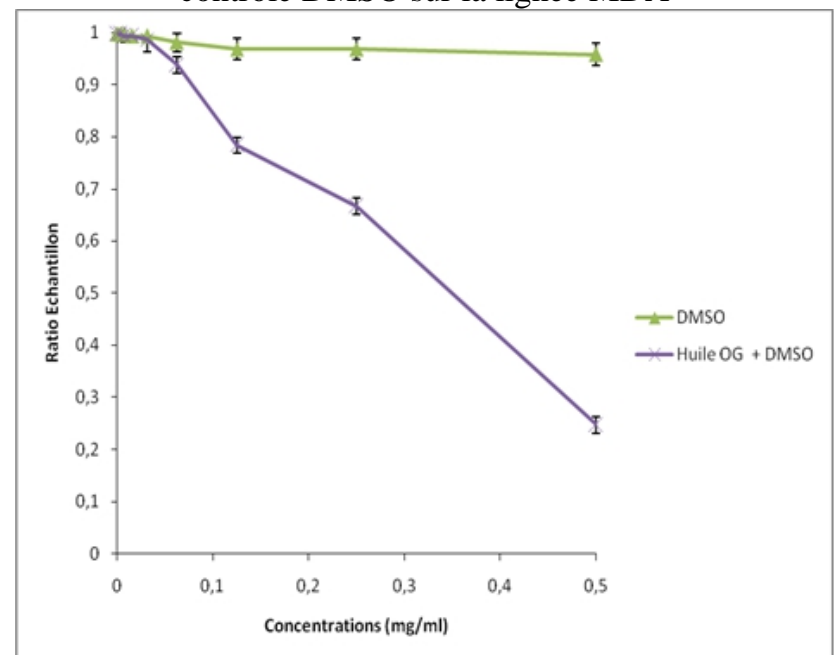

Figure 9: Effet cytotoxique dose-réponse de l'Huile essentielle OG solubilisé au DMSO et le contrôle DMSO sur la lignée U87. 
L'activité antioxydante de l'huile essentielle de Ocimum gratissimum à travers le test au DPPH a été évaluée à $\mathrm{F}=187 \pm 1,57 \mathrm{mM}$ Trolox $/ \mathrm{ml}$ ou $\mathrm{I}=38$ $\pm 0,74 \%$.

\section{Au niveau de l'activité antifongique}

Le nombre de colonies dans les tubes expérimentaux diminuait au fur et à mesure que les concentrations de l'Huile essentielle augmentaient. L'huile essentielle de $O$. gratissimum a inhibé la croissance des champignons cibles avec des valeurs de CMI variant de $12,50 \mu \mathrm{g} / \mathrm{ml}$ à $100 \mu \mathrm{g} / \mathrm{ml}$. A l'exception de la souche de Candida glabrata, les valeurs de CMF de l'huile essentielle sont identiques aux valeurs de CMI sur chacune des trois autres champignons cibles. Les plus basses CMI et CMF ont été obtenues à $12,50 \mu \mathrm{g} / \mathrm{ml} \operatorname{sur} C$. albicans, la plus forte CMI a été obtenue à $50 \mu \mathrm{g} / \mathrm{ml}$ sur $A$ fumigatus et $C$. glabrata alors que la plus forte CMF a été obtenue à $100 \mu \mathrm{g} / \mathrm{ml}$ sur $C$. glabrata. Les valeurs de CMI et CMF obtenues sont représentées par les histogrammes de la figure 10.

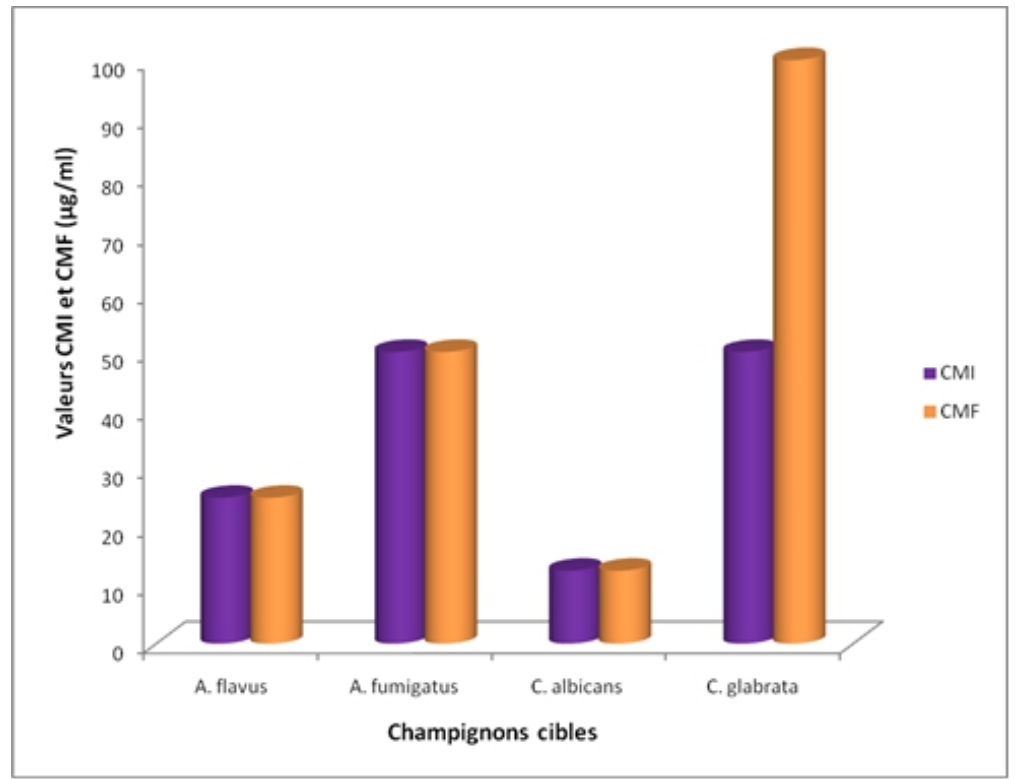

Figure 10: Histogrammes comparés des valeurs de CMI et CMF de l'Huile essentielle de Ocimum gratissimum

\section{Discussion}

\section{Au niveau des activités antitumorale et antioxydante}

La figure 3 est une illustration de l'effet cytotoxique observée de l'huile essentielle sur les cellules HCT 116. En présence de MTS, dans les puits contenant l'Huile essentielle à de fortes concentrations, on a observé une coloration différente de celle des puits contenant que du DMSO 1\%. 
Les figures 4 à 9 ont présenté des courbes à l'allure décroissante, cela indique que l'huile essentielle de Ocimum gratissimum a eu un effet cytotoxique dose dépendante sur les lignées cellulaires cibles. En présence de DMSO seul, les ratios calculés sont presque constants autour de 1, et même avec la plus grande concentration de DMSO, cela indique que le DMSO seul a été sans effet toxique sur les lignées cellulaires. Les valeurs de $\mathrm{CI}_{50}$ déterminées pour l'huile essentielle de $O$. gratissimum ont permis de distinguer que la lignée cellulaire Jurkat a été la plus sensible à l'huile essentielle car la valeur de la $\mathrm{CI}_{50}$ a été la plus basse $(80 \pm 0.02 \mu \mathrm{g} / \mathrm{ml})$ tandis que la lignée UB87 HTB-14 a été la moins sensible avec une $\mathrm{CI}_{50}$ la plus élevée $(200 \pm 0,02$ $\mu \mathrm{g} / \mathrm{ml})$. Les autres lignées cellulaires à savoir HCT 116, Hela, MDA-MDB231 et Ramos CRL 1596 dont les valeurs de $\mathrm{CI}_{50}$ sont situées entre les deux extrêmes pourraient être considérées comme moyennement sensibles. En considérant les critères du NCI (National Cancer Institute) mentionnés par Boyd (1997), l'huile essentielle de O. gratissimum serait faiblement active sur les lignées cellulaires cibles.

Les résultats de l'activité antioxydante de l'huile essentielle de $O$. gratissimum ont montré que cette huile est un puissant antioxydant car l'activité antioxydante a été évaluée à $\mathrm{F}=187 \pm 1,57 \mathrm{mM}$ Trolox $/ \mathrm{ml}$ ou $(\mathrm{I}=38$ $\pm 0,74 \%)$. Cette activité est nettement meilleure que celle obtenue dans les mêmes conditions expérimentales avec les extraits éthanolique et methanolique de Cymbopogon citratus respectivement à $\mathrm{F}=178.069 \pm 1,57$ $\mathrm{mM}$ Trolox $/ 1 \mathrm{ml}(\mathrm{I}=34 \pm 0,74 \%)$ et $\mathrm{F}=173,931 \pm 0,87 \mathrm{mM}$ Trolox $/ \mathrm{ml}$ ou ( $\mathrm{I}=33 \pm 0.26 \%$ ) (Kporou et $a l ., 2017$ ). Les activités antioxydantes des huiles essentielles provenant du genre Ocimum ont été bien décrites dans la littérature et ces activités sont fort intéressantes (Bozin et al., 2006; Pereira et Maia, 2007). A ce niveau, Owen et al., (2000) ont également rapporté que les extraits végétaux riches en composés phénoliques et présentant un pouvoir antioxydant très marqué pourraient jouer un rôle intéressant dans la prévention du cancer car ce sont des stabilisateurs des radicaux libres. L'huile essentielle de $O$. gratissimum pourrait donc être utilisée dans le domaine nutraceutiques en vue de réduire les risques de survenue de cancer. Les huiles essentielles agissent au niveau de la prévention du cancer ainsi qu'au niveau de sa suppression. Il est bien connu que certains aliments, comme l'ail ou le curcuma, sont de bonnes sources d'agents anticancéreux utiles pour prévenir l'apparition de cancer (Béliveau et al., 2006, Seifried, 2007).

\section{Au niveau de l'activité antifongique}

Dans les différents tubes expérimentaux, le nombre de colonies des champignons diminuait au fur et à mesure que les concentrations augmentaient dans les tubes, cela indique que l'huile essentielle de $O$. gratissimum a agi selon une relation dose réponse. Les valeurs de $\mathrm{CMI}$ et $\mathrm{CMF}$ obtenues sont 
représentées par les histogrammes de la figure 10. Sur chacune des souches de A. flavus , A. fumigatus et C. albicans, les valeurs des CMI et CMF ont été identiques, cela montre que sur ces souches l'huile $O$. gratissimum a eu un effet fongicide. Par contre, sur C. glabrata, l'huile a eu un effet fongistatique car les valeurs $\mathrm{CMI}$ et $\mathrm{CMF}$ ont été différentes. La plus faible valeur de $\mathrm{CMF}$ obtenue à $12,50 \mu \mathrm{g} / \mathrm{ml}$ sur $C$. albicans indique que cette souche a été la plus sensible à l'huile essentielle alors que la souche de $C$. glabrata a été la moins sensible car la $\mathrm{CMF}=100 \mu \mathrm{g} / \mathrm{ml}$ a été la plus élevée. Les souches de A. flavus et A. fumigatus ont une sensibilité intermédiaire entre les deux etrêmes. En outre, de façon générale, ces résultats ont fait ressortir que les moisissures $(A$. flavus et A. fumigatus) ciblées ont été plus sensibles à l'huile essentielle, car sur ces champignons l'huile a eu un effet fongicide contrairement aux levures (C. albicans et $C$. glabrata) pour lesquelles un effet fongistatique a été observé sur C. glabrata.

Des travaux menés par Oussou et al., (2004) ont également montré que cette huile a une activité sur la croissance in vitro d'une large gamme de bactéries dont les genres de Salmonella, Klebsiella, Citrobacter, Enterobacter, Pseudomonas, Staphylococcus etc..pour lesquels des effets bactéricides et bactériostatiques ont été observés en fonction de la bactérie.

Les activités biologiques observées avec l'huile essentielle de ocimum gratissimum seraient dues à la nature de la composition chimique de cette huile. En effet, des travaux antérieurement menée par Oussou et al., (2004) sur l'huile essentielle de cette espèce végétale récoltée en Côte d'Ivoire ont montré qu'elle est majoritairement riche en para-cymène, en $\gamma$-terpinène et en thymol. Il est de notoriété scientifique que le thymol est un composé phénolique ayant une puissante activité antimicrobienne d'ou son utilisation dans les bains de bouche (Lacoste et al., 1996; Pauli, 2001). La présence de ce composé dans cette huile essentielle justifierait en partie donc ses activités antioxydante, cytotoxique et antifongique observées au cours de cette étude. En effet, les composés phénoliques ont la capacité de bloquer la prolifération des cellules humaines issues de cancer (Edris, 2007; Braga et al., 2006, Cushine et al., 2005). Le thymol seul ne saurait justifier la diversité d'activités de cette huile car la présence des composés tels les alcools, les aldéhydes, les cétones monoterpéniques, les phenylpropanes et les monoterpènes pourraient agir par synergie ou addition d'effet, car ces composé sont connus pour leurs propriétés antimicrobiennes (Paster et al., 1995; Yang et al., 1996; Lahlou, 2004). Selon Oussou et $a l .$, (2004), les composés antibactériens et antifongiques contenus dans cette huile essentielle seraient d'environ 54,32\% par rapport à tous les composés identifiés. Ce résultat a été confirmé par les travaux de Kalemba et Kunicka, (2004), qui ont isolé et évalué séparément le potentiel antimicrobien des composés volatiles de certaines huiles essentielles. 


\section{Conclusion}

La présente étude a permis de mettre en évidence par des tests validés les activités cytotoxique, antioxydante et antifongique de l'huile essentielle de Ocimum gratissimum, une plante largement utilisée en médecine traditionnelle ivoirienne. L'activité cytotoxique en vue de la détermination du potentiel antitumoral a donné des valeurs de $\mathrm{CI}_{50}$ différentes selon la lignée cellulaire. L'huile essentielle a un effet cytotoxique dose-dépendante sur les lignées cellulaires Ramos CRL 1596, Jurkat, U87 HTB-14, HCT 116 CCL 47, MDAMB-231 et HeLa. La lignée cellulaire immunitaire Jurkat semble la plus sensible à cette huile. L'activité antioxydante de cette huile est intéressante. L'étude de l'activité antifongique a montré que cette huile a inhibé la croissance in vitro des souches de champignons testés. Cette huile a été fongicide sur les souches de Aspergillus flavus, Aspergillus fumigatus, Candida albicans mais fongistatique sur Candida glabrata. Du fait des propriétés biologiques intéressantes de cette huile, on pourrait envisager de développer des phytomédicaments standardisés et des nutraceutiques dont la production à partir de l'huile essentielle de O. gratissimum pourrrait respectivement contribuer à traiter les mycoses opportunistes et à réduire les risques de survenue de cancer.

\section{Remerciements}

Les remerciements sont adressés au Professeur Adela Pintea du Département de Chimie et Biochimie de L'Université des Sciences Agricoles et Médecine Vétérinaire, Cluj-Napoca, Roumanie pour sa contribution à la détermination de l'activité antioxydante de l'huile essentielle de cette plante.

\section{References:}

1. Ackah A. B. A. J., 2004. Spectre anti-infectieux de MISCA F3 sur la croissance in vitro de Candida albicans, Cryptococcus neoformans, Aspergillus flavus, Aspergillus fumigatus, Trichophyton mentagrophytes, Trichophyton rubrum. Mémoire de DEA de Biotechnologie, option Pharmacologie des substances naturelles, Université de Cocody Abidjan, 35 p.

2. Béliveau R. \& Gingras D., 2005. Les aliments contre le cancer. Édition du Trécarré, Outremont. 213 p.

3. Bouchet N., Barrier L. \& Fauconneau B., 1998. Radical scavenging activity and antioxydant property of tannins from Guiera senegalensis (Combretaceae), Phytoth. Res. 12: 159-162.

4. Boyd M. R., 1997. Preclinical screening, Clinical Trials, and Approval. in Anticancer Drug Development Guide, Teicher, B. A., Ed.; Humana Press. Totowa, NJ, pp. 23-42. 
5. Bozin B., Mimica-Dukic, N., Simin N. \& Anackov G., 2006. Characterization of the volatile composition of essential oils of some Lamiaceae spices and the antimi-crobial and antioxidant activities of the entire oils, J. Agric. Food Chem. 54: 1822-1828.

6. Braga P. C., Dal S. M., Culici M., Ga Sastri L., Marceca M. X. \& Guffanti E. E., 2006. Antioxidant potential of thymol determined by chemiluminescence inhibition in human neutrophils and cell-free systems, Pharm. 76: 61-68.

7. Butterfieid D. \& Lauderback C., 2002. Lipid peroxidation and protein oxidation in Alzheimer's disease brain: potential causes and consequences involving amyloid beta-peptide associated free radical oxidative stress, Fr. Rad.. Biol. Med. 32: 1050-1060.

8. Celso V. N., Tania U. N., Erika B., Abrahão F. N. M., Díogenes A. G. C. \& Benedito P. D. F., 1999. Antibacterial Activity of Ocimum gratissimum L. Essential Oil, Mem. Inst. Oswaldo Cruz 94 (5): 675678.

9. Cushnie T. P. \& Lamb A. J., 2005. Antimicrobial activity of flavonoids, Inter. Jour. Antimicrob. Agents. 26(5):343-356.

10. Djeneb C., Kouadio B., Goueh G., N'guessan B. Y. F., \& Guédé N. Z., 2016. Etude ethnobotanique, Evaluation de l'activité antifongique sur Candida albicans et de la toxicité sur des cellules Hff de Bersama Abyssinica (Fresen.), une Plante de la pharmacopée ivoirienne. Eur. Sc. Jour.12(3): 171-185.

11. Dzoyem J. P.,Tangmouo J. G., Manfouo J. R., Lontsi D., Etoa F. X. \& Lohouc P. J., 2006. Activité antifongique des extraits de quelques plantes médicinales CAMEROUNAISES, Nig. J. Nat. Prod. And Med. 10: 54-66.

12. Edris A. E., 2007. Pharmaceutical and therapeutic potentials of essential oils and their individual volatile constituents: A review, Phytoth. Res. 21: 308-323.

13. Gardner P., 1997. Superoxide-driven a conitase FE-S center cycling, Biosc. Rep. 17: 33-42.

14. Modzelewska A., Sur S., Kumar K. S. \& Khan S. R., 2005. Sesquiterpenes: Natural products that decease cancer growth., Curr. Med. Chem. Anti-cancer Agents 5: 477-499.

15. Heath H. B., 1981. Source Book of Flavours. Westport: Avi, 890 p.

16. Kalemba D. \& Kunicka A., 2004. Antibacterial and Antifungal Properties of Essential Oils, Curr. Med. Chem. 10: 813-829.

17. Kim K. S., Lee S., Lee Y. S., Jung S. H., Park Y., Shin K. H \& Kim B. K., 2003. Anti-oxidant activities of the extracts from the herbs of Artemisia apiacea, Jour. Ethnoph. 85: 69-72. 
18. Kohen R. \& Nyska A., 2002. Oxidation of biological systems: oxidative stress phenomena, antioxidants, redox reactions and methods for their quantification, Tox. Path. 30: 620-650.

19. Kouamé J. C., Gnoula E., Palé B. H., Guissou I. P., Simporé J. \& Nikiéma J., 2009. Etude des propriétés cytotoxiques et anti-radicalaires d'extraits de feuilles et de galles de Guiera senegalensis J. F. Gmel (Combretacae), Sc. et tech., Sc. santé 32(1): 9-23.

20. Kporou K. E., Kra A. K. M., Ouattara S. \& Guédé-Guina F., 2010. Évaluation de l'activité antifongique de Mitracarpus scaber, une rubiaceae codifiée MISCA sur Candida glabrata, Thér. 65: 1-4.

21. Kporou K. E., Coulibaly I., Rodica P., Pintea A., Ouattara S. \& Odagiu A., 2017. HPLC Phenolic Compounds Analysis and Antifungal Activity of extract's from Cymbopogon citratus (DC) Stapf against Fusarium graminearum and Fusarium oxysporum sp tulipae, Jour. Sc. Res. Rep. 15(1): 1-11.

22. Lacoste E., Chaumont J. P., Mandin D., Plumel M. M. \& Matos F. J. A.. 1996. Les proprietes antiseptiques de l'huile essentielle de Lippia sidoides Cham. Application a Ia microflore cutanee, Ann. Pharm. Franr. 54(5): 228-230.

23. Lahlou M., 2004. Methods to study phytochemistry and bioactivity of essential oils, Phytoth. Res. 18: 435-448.

24. Mashtaq A., Muhammad I. K., Muhammad R. K., Nawshad M. \& Amin U. K., 2013. Role of Medicinal Plants in Oxidative Stress and Cancer; Open Acc. Sci. Rep. (2)2: 1-3.

25. Newman D. J., Cragg G. M. \& Snader K. M., 2003, Natural Products as Sources of New Drugs over the Period 1981-2002, Jour. Nat. Prod. 66: 1022-1037.

26. Oussou K. R., Coffi K., Nathalie G., Séri Y., Gérard K., Mireille D.,Yao T. N., Gilles F. \& Jean-Claude C., 2004. Activités antibactériennes des huiles essentielles de trois plantes aromatiques de Côte d'Ivoire, C-R Chimie 7: 1081-1086.

27. Owen R. W., Giacosa A., Hull W. E., Haubner R., Spiegelhalder B. \& Bartsch H., 2000. The antioxidant/anticancer potential of phenolic compounds isolated from olive oil, Eur. Jour. Cancer 36 (10): 1235 1247.

28. Paster N., Menasheron M., Ravid U. \& Juven B., 1995. Antifungal activity of oregano and thyme essential oils applied as fumigants against fungi attacking stored grain, Jour. Food Prot. 8(1): 81-85.

29. Pauli A., 2001. Antimicrobial properties of essential oil constituents. Int. Jour. Aromather. 11: 126-133.

30. Rex J. H., Rinaldi M. G. \& Pfaller M. A., 1995. Resistance of Candida species to Fluconazole, Antimicrob. Agents Chemoth. 39: 1-8. 
31. Robert G., 2000. Les Sens du Parfum. Osman Eroylles Multimedia. Paris. 224 p.

32. Sanchez-Moreno C., 2002. Methods used to evaluate the free radical scavenging activity in foods and biological systems. Int. Jour. Foods Sc. Tech. 8: 121-137.

33. Seifried H. E., 2007. Oxidative stress and antioxidants: a link to disease and prevention? Jour. Nutr. Bioch. 18: 168-171.

34. Sheehan D. J., Hitchcock C. A. \& Sibley C. M., 1999. Current and emerging azole antifungal agents., Clin. Microb. Rev. 12(1): 40-79.

35. Valko M., Rhodes C. J., Moncol J., Izakovic M. \& Mazur M., 2006. Free radicals, metals and antioxidants in oxidative stress-induced cancer, Chemico-Biol. Inter. 160: 1-40.

36. Yang D., Michel D., Mandin D., Andriamboavonjy H., Poitry P.,. Chaumont J. P. \& Millet-Cierc J., 1996. Propriétes antifongiques et antibacteriennes in vitro de trois huiles essentielles de Patchouli d'origines differentes, Acta. Bot. Gallica 143(1): 29-35.

37. Young L. Y., Hull C. M. \& Heitman J., 2003. Distribution of ergosterol biosynthesis confers resistance to amphotericin B in Candida lusitaniae, Antimicrob. Agents Chemoth. 47: 2717-2724. 